\title{
Risk of Cancer in 767 Patients with Giant Cell Arteritis in Western Norway: A Retrospective Cohort with Matched Controls
}

\author{
Lene Kristin Brekke (D), Bjørg-Tilde Svanes Fevang, Andreas P. Diamantopoulos (D), \\ Jörg Assmus, Elisabet Esperø, and Clara Gram Gjesdal
}

ABSTRACT. Objective. To determine the risk of cancer in a large Norwegian cohort of patients with giant cell arteritis (GCA).

Methods. This is a hospital-based, retrospective, observational cohort study including patients diagnosed with GCA in the Bergen Health Area during 1972-2012. Patients were identified through computerized hospital records using the International Classification of Diseases coding system. Medical records were reviewed. Each patient was randomly assigned population controls matched on age, sex, and geography from the Central Population Registry of Norway. Data on the occurrence of cancer were obtained from the Cancer Registry of Norway. The cumulative risk of malignancy was estimated using Kaplan-Meier methods and potential differences were analyzed using the Gehan-Breslow and log-rank tests.

Results. We identified 881 cases with a clinical diagnosis of GCA, of which 792 fulfilled the American College of Rheumatology (ACR) 1990 classification criteria and 528 were biopsy-verified. Cases with no registered cancer prior to GCA diagnosis were included in a time-to-event analysis, with first cancer as the event ( $\mathrm{n}=767$ with clinical GCA diagnosis, 686 fulfilling ACR criteria for GCA, 463 biopsy-verified). These cases were matched with previously cancer-free population controls ( $\mathrm{n}=1437$, 1284,895 , respectively). We found no significant difference in the risk of malignancy after time of diagnosis/matching for GCA patients compared to population controls $(\mathrm{p}>0.05)$.

Conclusion. In this study of a large and well-characterized cohort of patients with GCA, there was no difference in the risk of malignancy in patients with GCA compared to matched population controls. (First Release January 15 2020; J Rheumatol 2020;47:722-9; doi:10.3899/jrheum.190147)

Key Indexing Terms:

VASCULITIS

GIANT CELL ARTERITIS

CANCER

TEMPORAL ARTERITIS
EPIDEMIOLOGY

MALIGNANCY

Giant cell arteritis (GCA) is the most common systemic vasculitis in adults. The immunopathogenesis of GCA, with

From the Hospital for Rheumatic Diseases, Haugesund; Department of Clinical Science, University of Bergen, Bergen; Bergen Group of Epidemiology and Biomarkers in Rheumatic Disease (BEaBIRD), Department of Rheumatology, and Centre for Clinical Research, Haukeland University Hospital, Bergen; Martina Hansens Hospital, Barum, Norway.

This work was supported by unrestricted research grants from the Norwegian Association of Heart and Lung Patients; The Norwegian Rheumatism Association; Marit Hansens Memorial Fund; Merck Sharp \& Dohme; Odd Fellow Medical Research Fund; and The Raagholt Foundation.

L.K. Brekke, MD, Hospital for Rheumatic Diseases, and Department of Clinical Science, University of Bergen; B.T. Fevang, $M D, P h D$, Department of Clinical Science, University of Bergen, and BEaBIRD, Department of Rheumatology, Haukeland University Hospital; A.P. Diamantopoulos, MD, PhD, MPH, Martina Hansens Hospital; J. Assmus, PhD, Centre for Clinical Research, Haukeland University Hospital; E. Esper $\phi, M D$, Hospital for Rheumatic Diseases; C. Gram Gjesdal, MD, PhD, Department of Clinical Science, University of Bergen, and BEaBIRD, Department of Rheumatology, Haukeland University Hospital.

Address correspondence to Dr. L.K. Brekke, HSR AS, PB 2175, 5504

Haugesund, Norway.E-mail: lene.kristin.brekke@hsr.as

Accepted for publication June 25, 2019. its complexity and still many unknown factors, has been outlined in a review by Weyand, et al and more recently by Hid Cadena, et $a l^{1,2}$. These reviews describe several lineages of dysfunctional immune cells as well as age-related remodeling of the immune system in GCA. The balance between the expression of coinhibitory and costimulatory immune checkpoint molecules appears to be crucial in fine-tuning the immune response and preventing autoimmunity. However, activation of specific inhibitory signals also allows cancer cells to avoid recognition and destruction ${ }^{3,4}$. Immune checkpoint inhibitors, used in cancer treatment, have been followed by a range of immune-related adverse events, including polymyalgia rheumatica (PMR) and large-vessel vasculitis $(\mathrm{LVV})^{5,6,7,8,9}$. The increasing use of these drugs, which generate antitumor activity but also enhance autoimmunity, draws new attention to the "old" question of whether there is an association between GCA and cancer. Several investigators have addressed this question, but published reports have shown conflicting results ${ }^{10-18}$. A metaanalysis from 2014 reported a low but statistically significant increased malignancy risk for patients with $\mathrm{GCA}^{19}$. However, most

Personal non-commercial use only. The Journal of Rheumatology Copyright $($ C 2020. All rights reserved. 
studies on the risk of cancer in GCA have been limited by small sample sizes, possible misclassification bias, and/or short periods of followup. We report a large cohort study in which GCA diagnoses have been thoroughly verified and cancer diagnoses were obtained from a national registry with mandatory reporting, providing virtually no loss to followup. This study may contribute to clarifying the association between GCA and the risk of malignancy.

\section{MATERIALS AND METHODS}

This is a retrospective, observational cohort study including patients diagnosed with GCA in the Bergen Health Area during 1972-2012. Our material represents a predominantly white referral cohort from mixed rural and urban areas. The study setting was the Bergen Health Area, consisting of 3 somatic hospitals: Haukeland University Hospital, Haraldsplass Deaconess Hospital, and Voss Hospital. Together these hospitals provide specialist healthcare services to the inhabitants of 22 municipalities in Hordaland County in western Norway. By population, Hordaland is the third largest county in Norway, and home to $10 \%$ of Norway's total population (Statistics Norway 2016). In this region there is only 1 laboratory for pathology, and no private hospitals that care for rheumatology patients. The majority (>90\%) of rheumatologists and internal medicine specialists in the area are hospital-based, with only 3 private rheumatologists in the region, all publicly funded and collaborating closely with the hospital departments. Patients were identified through hospital records using the International Classification of Diseases (ICD) coding system: ICD-8 (446.4) for 1972-1987, ICD-9 (446.5) for 1987-1998, and ICD-10 (M31.5-6) for 1999-2012. Electronic medical records of hospital diagnoses were available from 1972. Medical records that were completely electronic were available from 2001. For patients diagnosed in 1972 through 2001, we also obtained access to archived paper-based medical records to complete the extraction of clinical data. We note that cancer data were obtained separately, because this information was provided by the Cancer Registry of Norway (CRN), in which all new cases of cancer in Norway have been registered since 1952. We collected other data by reviewing medical records of every patient registered with the diagnosis of GCA following an outpatient visit or admission to any ward in one of the 3 study hospitals between January 1, 1972, and December 31, 2012 (41-yr period). We excluded patients if their GCA diagnosis originated prior to the beginning of our study, if data were unavailable, or if the review of records from the time of diagnosis concluded that GCA was an implausible diagnosis. We subclassified patients according to the American College of Rheumatology (ACR) 1990 criteria for GCA and according to temporal artery biopsy (TAB) result. Further details about the selection process including characteristics of the excluded cases have been published previously ${ }^{20}$. Every Norwegian is given a unique 11-digit identification number at birth or time of immigration. Each patient was initially matched for age (date of birth \pm 1 month), sex, and county of residence to 3 control subjects randomly selected from the Central Population Registry of Norway. The controls were required to be alive at the time of GCA diagnosis for their matched case and this date was defined as the start of the observation period for the control. The observation period ended with the diagnosis of cancer, death, or end of study (December 31,2012), whichever came first. We excluded duplicate control subjects, control subjects who were also among the cases, and all subjects with precancerous lesions or unverified malignancy (e.g., tumor with uncertain topography and/or unverified histopathology). For persons with $\geq 2$ registered cancers, we included only the first cancer. Only individuals with no registered cancer before the time of GCA diagnosis or corresponding date among matched controls (i.e., outcome-/event-free on entry to the cohort) were included in the time-to-event analysis. The original target of 3:1 matching was designed with the intent to analyze also cause-specific mortality, including rare causes of death. However, the high prevalence of cancer in the community created an imbalance in this matching, and to avoid unnecessary exclusion of previ- ously cancer-free cases, we dropped from 3:1 to 2:1 matching prior to the cancer-specific analyses. This was performed by random selection/inclusion of 2 of the original 3 controls when none of the controls had previous cancer. When only 1 of the 3 original controls had previous cancer, this control was excluded but the 2 cancer-free controls were kept in the analysis along with the cancer-free matched case. If 2 or all 3 original controls had previous cancer, we excluded all controls and the corresponding case from the time-to-event analysis.

Extensive demographic and clinical data were collected for the cases but for the population controls, we had no available information on potential risk factors, comorbid conditions, or other clinical data. Information on the occurrence of cancer was obtained from the $\mathrm{CRN}$. The $\mathrm{CRN}$ registration is based on mandatory reporting by physicians involved in the investigation, treatment, or followup of cancer patients in Norway. CRN also receives information about all cancer deaths registered by the Norwegian Cause of Death Registry. This ensures near-complete ascertainment of incident cancers in the Norwegian population and the CRN is among the oldest and most complete cancer registries in the world ${ }^{21}$. Every cancer diagnosis is required to be reported, except basal cell carcinoma (BCC) in adults. Precancerous lesions, benign tumors, and tumors with uncertain/unknown potential for malignancy are also reported. When the study was performed, the registration of cancers in CRN was complete for the entire study period. Variables received from the CRN include date and ICD code of the cancer diagnosis, and the diagnostic basis/accuracy of the malignancy. Cancer diagnoses were grouped according to the European Shortlist for Causes of Death, 2012 version (COD-SL-2012). The interpretation and reporting of these data are the sole responsibility of the authors, and no endorsement by the CRN is intended nor should be inferred. The study was approved by the REK sør- $\varnothing$ st B regional ethics committee (REC), which approved the study for all the hospitals involved (study reference number 2012/643/REK sør-øst B). REC granted permission to access records without obtaining consent from patients or their relatives owing to the long duration of the study and late onset of the disease. REC also granted permission to obtain a control cohort (3:1) matched on age, sex, and geography from the Central Population Registry of Norway.

Statistical analysis. Descriptive statistics were used to characterize the sample. The cumulative risk of cancer in cases and controls was estimated using Kaplan-Meier plots with the diagnosis of cancer as the event. P values comparing the Kaplan-Meier curves were calculated using the Gehan-Breslow and log-rank tests. Followup time was estimated using the reverse Kaplan-Meier method. HR were estimated using Cox regression with adjustment for time of diagnosis/matching (both linearly and categorized by decades). The significance level was set to 0.05 . The computing was done using the Statistical Package for the Social Sciences (SPSS) software version 24 (IBM Corp.) and R software version 3.4 (R Foundation for Statistical Computing).

\section{RESULTS}

We identified 881 patients with a clinical diagnosis of GCA, of whom 792 fulfilled the ACR 1990 classification criteria for GCA and 528 had biopsy-verified GCA based on TAB. Incidence numbers for GCA in the cohort were comparable to previous reports, with a mean annual cumulative incidence of 18.4 per 100,000 persons aged 50 years or more for clinically diagnosed GCA, 16.7 for cases fulfilling the ACR 1990 criteria, and 11.2 for biopsy-verified cases alone ${ }^{20}$. After excluding persons with registered cancer prior to GCA diagnosis, persons with registered cancer with uncertain diagnostic accuracy, controls that were also among the cases, and duplicate control subjects, we included the following numbers of cases and controls: 767 cases with a clinical diagnosis of GCA, of which 686 cases fulfilled the ACR 1990

Personal non-commercial use only. The Journal of Rheumatology Copyright (c) 2020. All rights reserved. 
criteria for GCA and 463 were biopsy-verified. These were matched (2:1) with $1437 / 1284 / 895$ previously cancer-free population controls, respectively. Core characteristics of the included cases and controls are presented in Table 1.

At the end of study (December 31, 2012), a total of $120 / 107 / 69$ patients with GCA and 227/204/141 population controls had been registered with a verified first malignancy in CRN after the time of GCA diagnosis/matching (Table 1). We found no significant difference in the risk of malignancy after time of diagnosis/matching for any subgroup of patients with GCA compared to population controls (Figure 1, Table 2). Followup times ranged from 0 to 35 years, with a median followup time of 3 years for cases and 5 years for controls. We did not compare the risk of cancer prior to GCA diagnosis/matching because of missing data on cancers diagnosed before 1972. Key features of our study compared to previous reports evaluating the risk of cancer in GCA cohorts from various populations are presented in Table 3 .

The overall distribution of various cancer types, grouped by codes in COD-SL-2012, showed no difference between cases and controls ( $p=0.768$; Figure 2$)$. The numbers for each cancer diagnosis are small and our study lacks sufficient power to analyze the risk of different cancer diagnoses separately. However, the combined lymphoid and hematopoietic malignancies (COD-SL-2012 codes 2.1.19-2.1.21) accounted for almost one-quarter of all registered cancers in both cases and controls in our study, afflicting 3.8\% of all GCA cases and $3.5 \%$ of their corresponding controls. Malignant neoplasms of the colon, rectum, and anus (COD-SL-2012 code 2.1.4) accounted for another quarter of the registered cancers in cases and about $20 \%$ of the registered cancers in controls. Cancers of the breast (COD-SL-2012 code 2.1.10) followed, accounting for 10.8\% of the registered malignancies in the patients with GCA and $12.8 \%$ in the controls.

\section{DISCUSSION}

This 41-year study of 881 Norwegian patients with GCA showed no difference in the risk of cancer compared to age-, sex-, and geographically matched population controls. This fits well with epidemiological data showing little or no difference in overall mortality rates of patients with GCA compared to the general population $22,23,24$. However, our result differs from that of some previous reports, in which an increased risk of cancer was seen in patients with GCA (Table 3).

Ungprasert, et al, in a metaanalysis from 2014, concluded with a low but statistically significant increased cancer risk, reporting a pooled risk ratio (RR) of 1.14 overall, 2.16 in the first 6-12 months after GCA diagnosis, and 1.35 for biopsy-proven $\mathrm{GCA}^{19}$. However, excluding a study with potential selection bias reduced the pooled RR to a nonsignificant level of 1.08. The excluded study was a large retrospective cohort study from Sweden reporting on 35,918 patients registered with a diagnostic code of GCA or PMR in a nationwide hospital discharge database ${ }^{16}$. The inclusion of only hospitalized patients may have led to a selection bias, favoring patients with severe disease who seem to have a higher cancer risk. A study by Michet, et al from the Mayo Clinic examined hospitalizations in the years 1996-2012 for patients with known GCA to evaluate whether hospitalization-related diagnoses accurately identified patients with this disease ${ }^{25}$. They found that the GCA diagnosis was mentioned in only $31 \%$ of 502 hospitalizations among patients with GCA. This illustrates the potential failure of identification that may bias any study based on hospitaladministrative diagnostic coding, ours included.

The highest risk of cancer in the Swedish study was noted during the first year after hospitalization for GCA or PMR ${ }^{16}$. This study was based solely on ICD coding without verification of the diagnosis through journal review. Consequently, incident cancers may have been misdiagnosed as GCA or PMR initially and thus contributed to the excess rate of cancer reported in the study. Symptoms similar to PMR and the sometimes-nonspecific presentation of GCA may mimic the presenting features of a variety of cancer diagnoses ${ }^{26-32}$. In the selection process for our study, $35 \%$ of the initially selected patients coded as GCA were excluded to ensure a cohort of correctly diagnosed $\mathrm{GCA}^{20}$. We verified GCA diagnoses by reviewing patient records from the time of diagnosis and we excluded patients if the reviewing rheuma-

Table 1. Core characteristics of cases and controls.

\begin{tabular}{|c|c|c|c|c|c|c|}
\hline \multirow[t]{2}{*}{ Characteristics } & \multicolumn{2}{|c|}{ Clinical Diagnosis } & \multicolumn{2}{|c|}{ ACR 1990 Criteria } & \multicolumn{2}{|c|}{ Biopsy-verified } \\
\hline & Cases, $n=767$ & Controls, $\mathrm{n}=1437$ & Cases, $\mathrm{n}=686$ & Controls, $\mathrm{n}=1284$ & Cases, $n=463$ & Controls, $\mathrm{n}=895$ \\
\hline \multicolumn{7}{|c|}{ Mean age at time of GCA diagnosis/ } \\
\hline Female sex, $\mathrm{n}(\%)$ & $551(71.8)$ & $1030(71.7)$ & $497(72.4)$ & $928(72.3)$ & $339(73.2)$ & $656(73.3)$ \\
\hline \multicolumn{7}{|l|}{ Median observation } \\
\hline time*, yrs $(95 \% \mathrm{CI})$ & $3(2-3)$ & $5(4-5)$ & $3(2-3)$ & $4(3-4)$ & $3(1-3)$ & $5(4-5)$ \\
\hline
\end{tabular}

* Observation time according to reverse Kaplan-Meier. ACR: American College of Rheumatology; GCA: giant cell arteritis.

Personal non-commercial use only. The Journal of Rheumatology Copyright $\odot$ 2020. All rights reserved. 

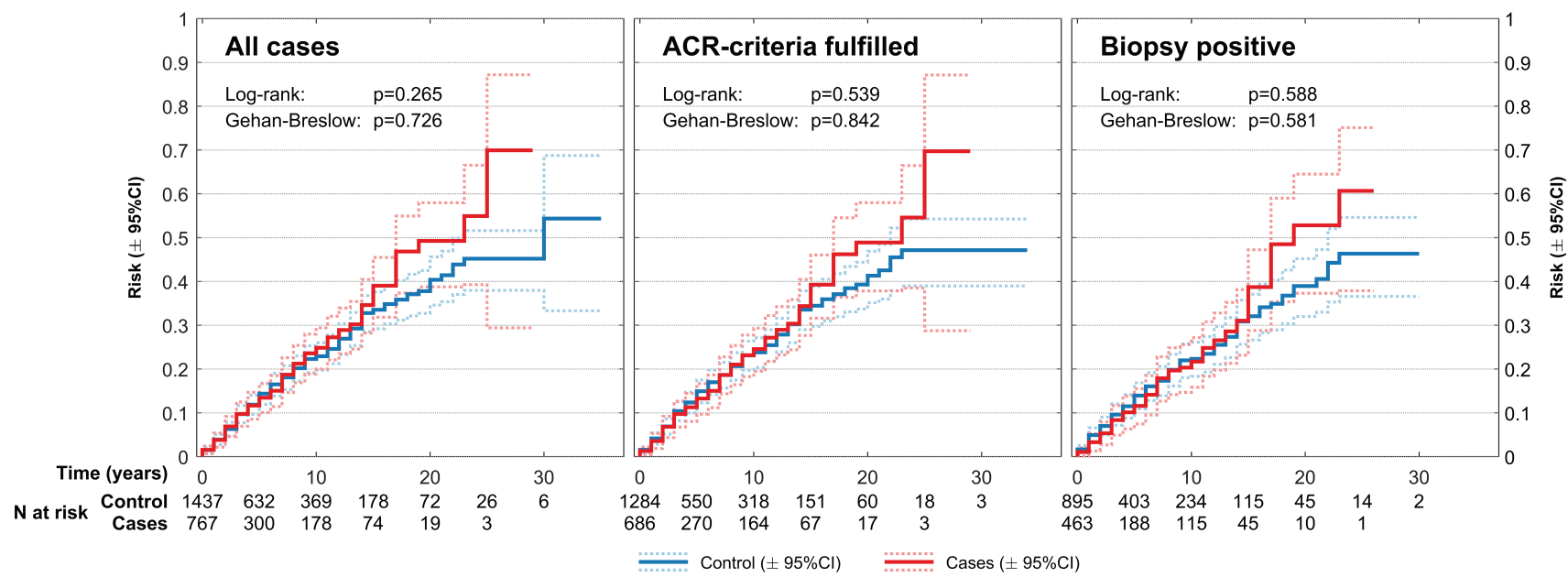

Figure 1. Cumulative risk of first malignancy after time of GCA diagnosis or matching. GCA: giant cell arteritis; ACR: American College of Rheumatology.

(A) All cases

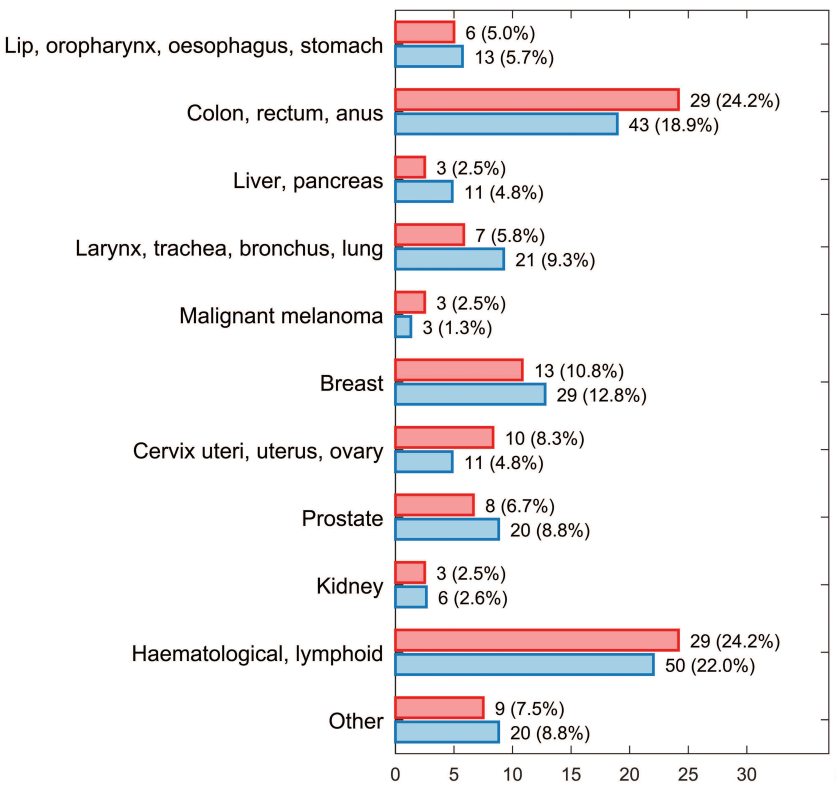

(B) ACR-criteria fulfilled

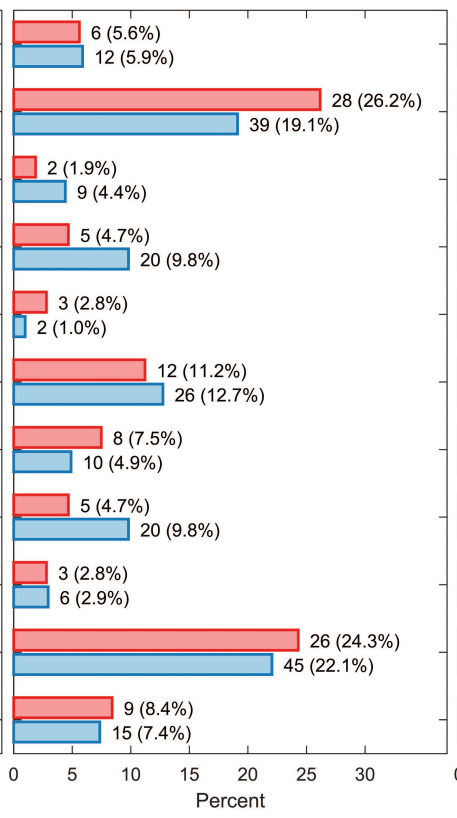

(C) Biopsy positive

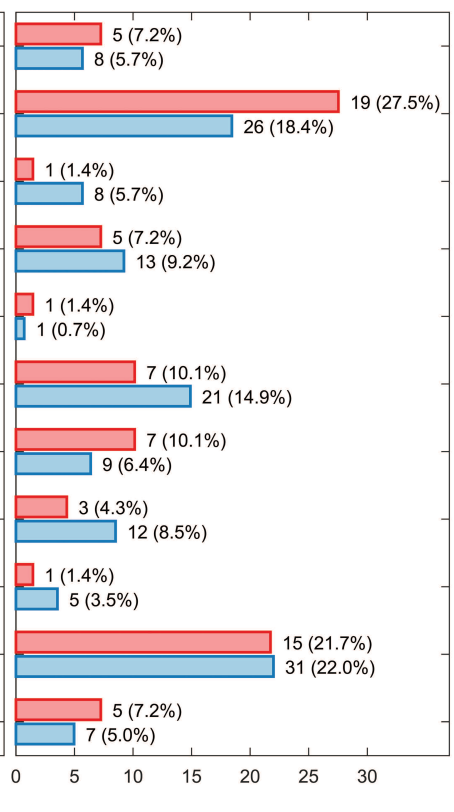

Controls

Figure 2. The distribution of different cancers in patients with GCA and matched controls in the Bergen Health Area (1972-2012). All values represent the number (\%) of persons with registered cancer. A. Incident cancers for 767 patients with clinically diagnosed GCA and 1437 matched controls. B. Incident cancers for 686 patients with GCA diagnosis fulfilling the ACR 1990 classification criteria for GCA and their 1284 matched controls. C. Incident cancers for 463 biopsy-proven patients with GCA and 895 matched controls. Cancers are grouped according to COD-SL-2012: lip, oropharynx, esophagus, stomach (codes 2.1.1-3); colon, rectum, anus (code 2.1.4); liver, pancreas (codes 2.1.5-6); larynx, trachea, bronchus, lung (codes 2.1.7-8); malignant melanoma of skin (code 2.1.9); breast (code 2.1.10); cervix uteri, uterus, ovary (codes 2.1.11-13); prostate (code 2.1.14); kidney (code 2.1.15); hematological, lymphoid (codes 2.1.19-21); and other (codes 2.1.16-18, 2.1.22). ACR: American College of Rheumatology; GCA: giant cell arteritis; COD-SL-2012: European Shortlist for Causes of Death (2012 version).

tologist concluded that GCA was an implausible diagnosis based on clinical information available at that time. Characteristics of the excluded cases have been published previously ${ }^{20}$. A similar proportion of erroneous diagnostic coding in the Swedish hospital discharge study would represent a significant limitation of their results.

The excellent completeness of the CRN ensures that nearly all incident cancers are registered ${ }^{21}$. A study conducted

\section{Personal non-commercial use only. The Journal of Rheumatology Copyright @ 2020 . All rights reserved.}


Table 2. HR for the risk of cancer in patients with GCA versus population controls

\begin{tabular}{lccc}
\hline & HR & Lower CI & Upper CI \\
\hline All Cases, $\mathrm{n}=2204$ & & & \\
$\quad$ Unadjusted & 1.13 & 0.91 & 1.42 \\
$\quad$ Adjusted for time of diagnosis/matching & 1.16 & 0.93 & 1.45 \\
$\quad$ Adjusted for time period (decades*) & 1.16 & 0.93 & 1.45 \\
ACR 1990 criteria fulfilled, $\mathrm{n}=1970$ & & 0.85 & 1.36 \\
$\quad$ Unadjusted & 1.12 & 0.87 & 1.39 \\
$\quad$ Adjusted for time of diagnosis/matching & 1.12 & 0.87 & 1.39 \\
$\quad$ Adjusted for time period (decades*) & 1.12 & 0.81 & 1.45 \\
Biopsy-positive, $\mathrm{n}=1358$ & & 0.84 & 1.49 \\
$\quad$ Unadjusted & 1.09 & 0.83 & \\
$\quad$ Adjusted for time of diagnosis/matching & 1.12 & & \\
$\quad$ Adjusted for time period (decades*) & 1.11 & & \\
\hline
\end{tabular}

* Decades defined as the time periods 1972-1982, 1983-1992, 1993-2002, and 2003-2012. ACR: American

College of Rheumatology; GCA: giant cell arteritis.

in a population where registration of cancers is not mandatory might be biased by an increased registration of cancers in patients followed within specialist care, compared to previously healthy individuals. Being monitored for a chronic disease might thus increase the probability of a subsequent cancer being registered. If so, this could partly explain the increased risk of cancer noted in patients with GCA in some studies.

To date no definite association between GCA/PMR and cancer has been agreed on because of conflicting epidemiological evidence, as illustrated in Table 3 . The majority of previous studies have been retrospective cohort studies. However, 2 of the studies had a prospective design although differently executed ${ }^{11,18}$. Prospective design is often considered superior as it allows an evaluation of the temporal sequence between exposure and outcome, and possibly reduces the risk of loss to followup. However, prospective studies are cost- and time-consuming, and often difficult to maintain for a long observation period, making them less suitable for the study of seldom and potentially late outcomes such as malignant diseases. Also, patients included in a prospective study are regularly evaluated and more likely to undergo extensive medical investigations, possibly leading to earlier detection and even an increased risk of cancer. As such, prospective studies run the risk of overestimating the incidence of cancer in the patient population compared to the controls. In the smaller prospective study, they found a standardized incidence ratio of malignancy of $4.61^{18}$. However, the number of patients was rather low $(n=107)$ and the number of recent and successive cancers was low $(n=7)$, rendering these numbers uncertain. Also, there were no age- and sex-matched controls, only the use of crude incidence rates.

It may be argued that a surveillance bias applies to any patient with GCA, often presenting with elevated inflammatory markers and nonspecific symptoms and signs. This clinical presentation may start an extensive diagnostic inves- tigation, in which concomitant cancers are detected earlier than they otherwise would have been, thus shifting the timing of cancer diagnosis from post-GCA to pre-GCA. However, a large population-based study from the UK found no difference in the frequency of prior cancer between patients with and without $\mathrm{GCA}^{17}$, and Kermani, et al found that patients with GCA in a cohort from Olmsted County (USA) had significantly fewer malignancies prior to diagnosis compared to controls ${ }^{33}$.

The 2 previously published reports on cancer risk in Norwegian patients with GCA concluded differently ${ }^{10,11}$. Myklebust, et al concluded that there was no difference in risk in their cohort of 398 patients with GCA and 1592 controls ${ }^{11}$. Haga, et al published a case-control study based on prospectively included cases of GCA or PMR during 1978-1983 with subsequent retrospective analysis of cancer risk based on cancer (registered in the CRN) from the inception of the registry through $1988^{10}$. The inclusion criteria were biopsy-proven GCA or fulfillment of study-specific clinical criteria for either GCA or PMR, and 185 cases and 925 controls were included (Table 3). While Haga, et al found no difference in overall cancer risk, they found an increased risk in biopsy-proven GCA with HR of 2.35 for cases versus controls. They also reported an increased HR of 7.25 for biopsy-positive compared to biopsy-negative cases. The Haga study included patients from hospitals within our catchment area and within our study period. However, they also included isolated PMR (91 of the 185 cases) and the study-specific inclusion criteria were somewhat different from ours. Further, the number of biopsy-proven GCA was low $(n=65)$, and the finding of 16 cancers among these might have been coincidental because we found no such difference in our 528 biopsy-proven cases. Two other previously published reports limited to biopsy-proven GCA cases have not confirmed the finding of increased risk associated with positive biopsy. In disagreement with Haga, et $a l^{10}$, they found no overall difference in

Personal non-commercial use only. The Journal of Rheumatology Copyright $\subset$ 2020. All rights reserved 


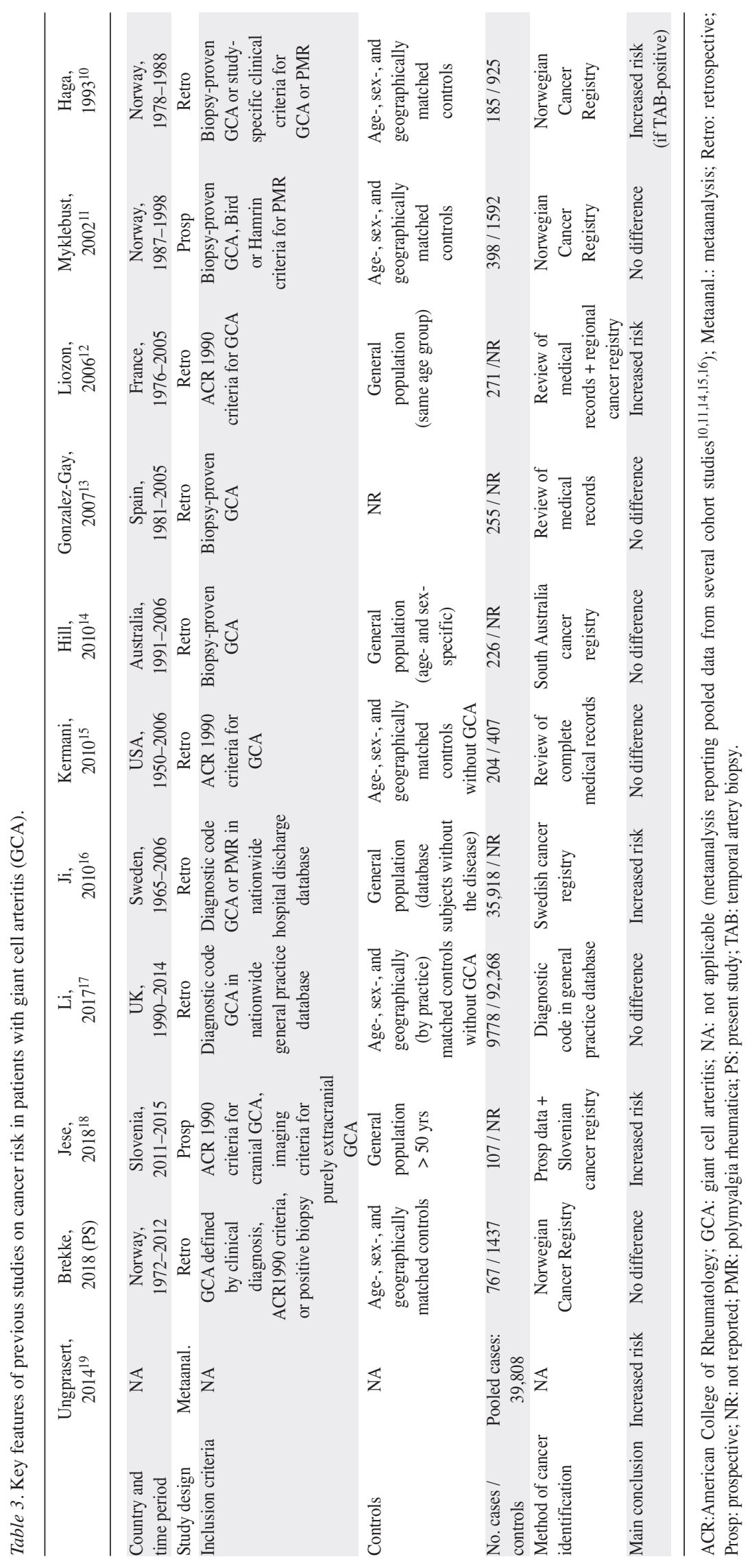

Personal non-commercial use only. The Journal of Rheumatology Copyright $($ C 2020. All rights reserved. 
risk of malignancy in biopsy-proven GCA compared to the general population $^{13,14}$.

Published reports have varied regarding the distribution of different types of cancers that patients with GCA experience. Kermani, et al found that nonmelanoma skin cancers were the most common cancers in both cases and controls ${ }^{15}$. This finding has not been confirmed by other studies, although $\mathrm{Ji}$, et al also found an excess of skin cancers $^{16}$. However, several studies have not included BCC of the skin as a malignancy ${ }^{10,11,12,14}$. BCC in adults are not reported to the $\mathrm{CRN}$ and were thus not included among cancers observed in our study or in the previous Norwegian studies.

Liozon, et al found a strong temporal association between GCA and myelodysplastic syndromes and reported a concurrent (occurring 1 yr before or after GCA diagnosis) hematological malignancy in $3.6 \%$ of their cases ${ }^{12}$. The combined malignancies of lymphoid and hematopoietic tissues accounted for almost one-quarter of the registered cancers in our study, afflicting $3.8 \%$ of all GCA cases and $3.5 \%$ of the matched controls. Overall, both frequency and types of cancers found in patients with GCA and population controls in our study were similar. This is in agreement with the findings reported by Myklebust, et al and largely with the sizeable Swedish study, except for their finding of more skin cancers and leukemia ${ }^{11,16}$. The numbers for each cancer diagnosis are small and most studies lack sufficient power to analyze the risk of different cancer diagnoses separately.

We have discussed factors that may have contributed to our findings being different from those of some other studies. Our data are limited by the retrospective design and have incomplete (for cases) and missing (for controls) data on important cancer risk factors such as smoking, use of cytotoxic medications or hormones, and other potential confounders such as body mass index and family history of malignancy. For example, if the prevalence of smokers is reduced in patients with GCA compared to the controls, our study could underestimate the true cancer risk associated with GCA. We also note that our cohort consists of cases with predominantly cranial GCA ( $65 \%$ with positive TAB). Thus, our results may not be representative for cases with purely extracranial LVV. One strength of our study is the completeness and high quality of the CRN. It has been mandatory to register every new case of cancer in Norway in this registry since 1952 . Another strength is the large and well-defined cohort of GCA cases resulting from a thorough review of clinical data, excluding misclassified cases, and including hospitalized patients, as well as those only treated in outpatient clinics. The study also included a large cohort of population controls that were tightly matched regarding the most significant of all cancer risk factors - age. The large sample size of both cases and controls rendered a well-powered analysis, allowing us to detect relevant differences between the groups. The long duration of our study (41 yrs) is a strength that reduces the risk of missing late-occurring cancers as well as diminishes the risk of erroneous conclusion based on variations through time. However, the long duration also poses a challenge because the diagnostic criteria for both cancer and GCA might have changed substantially during such a long period. Even so, time-dependent changes are controlled for by including the control population on which such factors presumably would have a similar effect.

Based on our findings, patients with GCA are not at increased risk of cancer following the diagnosis of their vasculitis. From a clinical point of view, our results indicate that patients with an unequivocal diagnosis of GCA do not need specific screening for malignancy.

\section{ACKNOWLEDGMENT}

The authors thank Dr. Rabab Adam, MD, for contributions in the early phase of the study. The authors also thank Alf Aksland (IT consultant, Haukeland University Hospital), Anita Mellingen (Department of Rheumatology, Haukeland University Hospital), Bjørg Sandvik (Department of Pathology, Haukeland University Hospital), Robinson Lazcano (Central archive, Haukeland University Hospital), Lisbeth Thorsen (Haraldsplass Deaconess Hospital), Laila Kvåle (Voss Hospital), and their coworkers, who aided with the identification of patients or the extensive retrieval of patient records.

\section{REFERENCES}

1. Weyand CM, Liao YJ, Goronzy JJ. The immunopathology of giant cell arteritis: diagnostic and therapeutic implications. J Neuroophthalmol 2012;32:259-65.

2. Hid Cadena R, Abdulahad WH, Hospers GA, Wind TT, Boots AM, Heeringa $\mathrm{P}$, et al. Checks and balances in autoimmune vasculitis. Front Immunol 2018;9:315.

3. Calabrese LH. Sorting out the complexities of autoimmunity and checkpoint inhibitors: not so easy. Ann Intern Med 2018; 168:149-50.

4. Calabrese L, Velcheti V. Checkpoint immunotherapy: good for cancer therapy, bad for rheumatic diseases. Ann Rheum Dis 2017;76:1-3

5. Goldstein BL, Gedmintas L, Todd DJ. Drug-associated polymyalgia rheumatica/giant cell arteritis occurring in two patients after treatment with ipilimumab, an antagonist of CTLA-4. Arthritis Rheumatol 2014;66:768-9.

6. Calabrese C, Kirchner E, Kontzias K, Velcheti V, Calabrese LH. Rheumatic immune-related adverse events of checkpoint therapy for cancer: case series of a new nosological entity. RMD Open 2017;3:e000412.

7. Hodi FS, Lawrence D, Lezcano C, Wu X, Zhou J, Sasada T, et al. Bevacizumab plus ipilimumab in patients with metastatic melanoma. Cancer Immunol Res 2014;2:632-42.

8. Micaily I, Chernoff M. An unknown reaction to pembrolizumab: giant cell arteritis. Ann Oncol 2017;28:2621-2.

9. Kuswanto WF, MacFarlane LA, Gedmintas L, Mulloy A, Choueiri TK, Bermas BL. Rheumatologic symptoms in oncologic patients on PD-1 inhibitors. Semin Arthritis Rheum 2018;47:907-10.

10. Haga HJ, Eide GE, Brun J, Johansen A, Langmark F. Cancer in association with polymyalgia rheumatica and temporal arteritis. J Rheumatol 1993;20:1335-9.

11. Myklebust G, Wilsgaard T, Jacobsen BK, Gran JT. No increased frequency of malignant neoplasms in polymyalgia rheumatica and temporal arteritis. A prospective longitudinal study of 398 cases and matched population controls. J Rheumatol 2002;29:2143-7.

Personal non-commercial use only. The Journal of Rheumatology Copyright (C) 2020. All rights reserved 
12. Liozon E, Loustaud V, Fauchais AL, Soria P, Ly K, Ouattara B, et al. Concurrent temporal (giant cell) arteritis and malignancy: report of 20 patients with review of the literature. J Rheumatol 2006; 33:1606-14.

13. Gonzalez-Gay MA, Lopez-Diaz MJ, Martinez-Lado L, Pena-Sagredo JL, Lopez-Agreda H, Miranda-Filloy JA, et al. Cancer in biopsy-proven giant cell arteritis. A population-based study. Semin Arthritis Rheum 2007;37:156-63.

14. Hill CL, Cole A, Rischmueller M, Dodd T, Coleman M, Tucker G, et al. Risk of cancer in patients with biopsy-proven giant cell arteritis. Rheumatology 2010;49:756-9.

15. Kermani TA, Schafer VS, Crowson CS, Hunder GG, Gabriel SE, Ytterberg SR, et al. Malignancy risk in patients with giant cell arteritis: a population-based cohort study. Arthritis Care Res 2010;62:149-54

16. Ji J, Liu X, Sundquist K, Sundquist J, Hemminki K. Cancer risk in patients hospitalized with polymyalgia rheumatica and giant cell arteritis: a follow-up study in Sweden. Rheumatology 2010;49:1158-63.

17. Li L, Neogi T, Jick S. Giant cell arteritis and vascular disease-risk factors and outcomes: a cohort study using UK Clinical Practice Research Datalink. Rheumatology 2017;56:753-62.

18. Ješe R, Rotar Ž, Tomšič M, Hočevar A. Giant cell arteritis and malignancy-more than just a coincidence? J Clin Rheumatol 2018;24:85-6.

19. Ungprasert P, Sanguankeo A, Upala S, Knight EL. Risk of malignancy in patients with giant cell arteritis and polymyalgia rheumatica: a systematic review and meta-analysis. Semin Arthritis Rheum 2014;44:366-70.

20. Brekke LK, Diamantopoulos AP, Fevang BT, Assmus J, Espero E, Gjesdal CG. Incidence of giant cell arteritis in Western Norway 1972-2012: a retrospective cohort study. Arthritis Res Ther 2017;19:278.

21. Larsen IK, Smastuen M, Johannesen TB, Langmark F, Parkin DM, Bray F, et al. Data quality at the Cancer Registry of Norway: an overview of comparability, completeness, validity and timeliness. Eur J Cancer 2009;45:1218-31.
22. Lee YH, Song GG. Overall and cause-specific mortality in giant cell arteritis: a meta-analysis. Z Rheumatol 2018;77:946-51.

23. Catanoso M, Macchioni P, Boiardi L, Muratore F, Restuccia G, Cavazza A, et al. Incidence, prevalence, and survival of biopsy-proven giant cell arteritis in Northern Italy during a 26-year period. Arthritis Care Res 2017;69:430-8.

24. Mohammad AJ, Nilsson JA, Jacobsson LT, Merkel PA, Turesson C. Incidence and mortality rates of biopsy-proven giant cell arteritis in southern Sweden. Ann Rheum Dis 2015;74:993-7.

25. Michet CJ 3rd, Crowson CS, Achenbach SJ, Matteson EL. The detection of rheumatic disease through hospital diagnoses with examples of rheumatoid arthritis and giant cell arteritis: what are we missing? J Rheumatol 2015;42:2071-4.

26. Aguiar T, Vincent MB. Giant cell arteritis and polymyalgia rheumatica as first manifestation of typical pulmonary carcinoid tumor. Reumatismo 2015;67:165-8.

27. Bhatti MT, Furman J, Gupta S, Tabandeh H, Monshizadeh R. Superficial temporal artery biopsy diagnostic for lung carcinoma. Am J Ophthalmol 2001;132:135-8.

28. Andersen SA, Kiss K. Primary temporal region squamous cell carcinoma diagnosed by a superficial temporal artery biopsy. Eur Ann Otorhinolaryngol Head Neck Dis 2015;132:91-2.

29. Masood I, While B, Mudhar HS. Perivascular mantle cell lymphoma affecting a temporal artery - a highly unusual cause of temporal headache. Cardiovasc Pathol 2011;20:244-6.

30. Linxweiler M, Hasenfus A, Wolf G, Schick B. Perivascular marginal zone lymphoma mimicking temporal arteritis. Otolaryngol Head Neck Surg 2015;152:187-8.

31. Naschitz JE, Slobodin G, Yeshurun D, Rozenbaum M, Rosner I. Atypical polymyalgia rheumatica as a presentation of metastatic cancer. Arch Intern Med 1997;157:2381.

32. Jalava-Karvinen P, Kemppainen J, Saario R, Kotilainen P. Metastasis in the temporal bone mimicking temporal arteritis. J Clin Rheumatol 2010;16:19-21.

33. Kermani TA, Schafer VS, Crowson CS, Hunder GG, Ytterberg SR, Matteson EL, et al. Cancer preceding giant cell arteritis: a case-control study. Arthritis Rheum 2010;62:1763-9. 